\title{
Electrospinning of uniform nanofibers of Polymers of Intrinsic Microporosity (PIM-1): The influence of solution conductivity and relative humidity
}

\author{
Fuat Topuz ${ }^{\mathrm{a}, *}$, Bekir Satilmis ${ }^{\mathrm{a}, \mathrm{b}, * *}$, Tamer Uyar $^{\mathrm{c}, * * *}$ \\ a Institute of Materials Science \& Nanotechnology, Bilkent University, Ankara, 06800, Turkey \\ ${ }^{\mathrm{b}}$ Department of Chemistry, Faculty of Arts and Sciences, Kirsehir Ahi Evran University, Kirsehir, 40100, Turkey

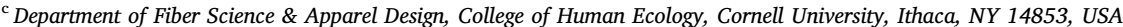

\section{H I G H L I G H T S}

- The conductivity of PIM-1 solutions was significantly increased by salt addition.

- Higher salt content drastically improved the electrospinnability of PIM-1 solutions.

- Very thin PIM-1 fibers with $160 \mathrm{~nm}$ in diameter could be produced by salt addition.

- Increasing relative humidity did not influence the electrospinnability of PIM-1.

- Less fibers were formed at very high humidity conditions $(\sim 80 \%)$ in a given time.

\section{A R T I C L E I N F O}

\section{Keywords:}

Polymers of Intrinsic Microporosity (PIMs)

Electrospinning

Fibers

Conductivity

Viscosity

\begin{abstract}
A B S T R A C T
Polymers of Intrinsic Microporosity (PIMs) are ultra-permeable macromolecules, which can be cast as a dense membrane and exploited in a wide spectrum of applications, particularly for gas separation owing to their extremely large inner surface area, free volume and high gas permeability. While they are mostly intended to serve as membranes for gas separation, in recent years, they have been also employed in water treatment applications owing to their solution processability, which enables the production of fibrous membranes by electrospinning. The fibrous form provides an increase in sorption performance, water permeability and flux for their application in water treatment. However, owing to the low conductivity of PIM-1 solutions in 1,1,2,2-tetrachloroethane (TeCA) that is the ideal solvent for the electrospinning of PIM-1 solutions, a higher polymer concentration is required to produce bead-free fibers. Furthermore, the electrospinning of highly concentrated PIM-1 solutions leads to the formation of microfibers. To tackle these problems, we herein incorporated an ammonium salt (i.e., tetraethylammonium bromide, TEAB) to increase the conductivity of PIM-1 solutions and study the impact of solution conductivity on the electrospinning of PIM-1 solutions. In parallel to the conductivity study, the influence of relative humidity on the electrospinning and morphology of PIM-1 fibers was explored. The addition of TEAB significantly increased the solution conductivity and drastically enhanced the electrospinnability of PIM-1. The electrospinning of PIM-1 solutions (10\% (w/v)) in the presence $7.5 \mathrm{wt} \% \mathrm{TEAB}$ (with respect to PIM-1) led to bead-free fibers, while at the same concentration, electrosprayed beads and droplet splashing were observed in the absence of TEAB. On the other hand, increasing humidity did not influence the electrospinnability of PIM-1 and the fiber texture, however, less fibers were formed in a given time at very high humidity conditions ( $\sim 80 \%)$. Overall, the experimental findings revealed that the addition of the salt drastically enhanced the electrospinnability of PIM-1 solutions owing to the enhanced conductivity and could lead to the formation of very thin PIM-1 fibers with $160 \mathrm{~nm}$ in diameter while no significant effect of relative humidity on the electrospinnability of PIM-1 solutions was observed.
\end{abstract}

\footnotetext{
*Corresponding author.

** Corresponding author. Institute of Materials Science \& Nanotechnology, Bilkent University, Ankara, 06800, Turkey.

**** Corresponding author.

E-mail addresses: fuat.topuz@rwth-aachen.de (F. Topuz), bekir.satilmis@ahievran.edu.tr (B. Satilmis), tu46@cornell.edu (T. Uyar).
} 


\section{Introduction}

There is an increasing demand in highly porous and ultra-permeable materials with enhanced sorption performance for rapid removal of pollutants by hydrophobic molecular microcavities available in the matrix [1-5]. Particularly, the use of polymers with very large surface areas allows rapid diffusion of pollutants through an inter-connected porous matrix for quick and efficient scavenging of water and air contaminants. In this regard, Polymers of Intrinsic Microporosity (PIMs) as a class of porous organic polymers can meet these expectations by virtue of their highly porous structure with very large surface areas $\left(>500 \mathrm{~m}^{2} \mathrm{~g}^{-1}\right.$ ) [6-10]. PIMs are composed of fused rings linked by the sites of contortion to render the resultant structure as rigid and highly non-linear as a result of inefficient packing of macromolecular chains $[10,11]$. The materials formed by PIMs can thus offer high solvent permeability and enhanced flux values [12]. Among various PIM derivatives, PIM-1 with a surface area of $740-850 \mathrm{~m}^{2} \mathrm{~g}^{-1}$ has been the subject of many studies with further advantages of facile synthesis and well-documented properties in various forms, including electrospun fibers $[13,14]$.

The electrospinning of PIM-1 fibers has been reported by different research groups for their use in water treatment for the scavenging of dyes [15,16], energy storage [17], volatile organic compounds [13] and nitrophenol reduction [18]. PIM-1 could successfully be electrospun from its solution in 1,1,2,2-tetrachloroethane (TeCA), which led to the bead-free microfibers of PIM-1 [15,17-19]. On the other hand, the solutions of PIM-1 in TeCA have very low conductivity, and therefore, the electrospinning of PIM-1 solutions requires a relatively high concentration to eject a single continuous fiber [13]. Since electrospinning is a fiber formation process that relies on the formation of a jet once the repulsion forces dominate the surface tension of the solution, many process and solution parameters become prominent factors on the fiber formation. The electrospinnability of a polymer solution is highly dependent on the solution properties, particularly viscosity [20], molecular weight [21], surface tension [22] and conductivity [23]. These factors are highly important in determining the properties of the resultant fibers in terms of morphology, size, and texture. Generally, the solution viscosity increases with a higher polymer concentration to generate a uniform fiber structure. However, this increase in viscosity leads to the formation of thicker fibers which is not desired in most applications. Electrospinnability can be improved by the incorporation of additives to enable bead-free fiber formation at lower polymer concentrations. In one example, Chen et al. improved the electrospinnability of the low concentrated poly(methyl methacrylate) solutions with the incorporation of hyperbranched poly(ester amine) (PEA) [24]. While bead formation observed in the absence of the additive, the incorporation of $1 \mathrm{wt} \%$ PEA led to bead-free fiber structure as a result of increased solution conductivity. On the other hand, no significant change in the viscosity and surface tension was observed in the respective solutions. Wang et al. showed the improved electrospinnability of low molecular weight poly(4-vinylpyridine) polymers by supramolecular interactions with 4,4'-biphenol or 4-hydroxy-4'-biphenyl carboxylic acid/ $\mathrm{ZnCl}_{2}$ to create hydrogen bonds and coordination interactions in the matrix [25]. Likewise, the use of salt (i.e., $\mathrm{NaCl}$ ) as an additive has shown a drastic improvement in the electrospinnability of poly(ethylene oxide) [26]. The influence of salt addition and solution conductivity on the electrospinning was also explored for various polymers, including polystyrene [23], poly(L-lactic acid) [27,28], poly (ethylene oxide) [29], polyamide-6 [30], poly(e-caprolactone) [31] and poly(acrylic acid) [32], and the authors observed drastic differences in the properties of the resultant fibers. In most cases, increasing conductivity produced thinner fibers due to the enhanced elongation level of a jet by an electrical force: higher conductivity may give rise to higher elongation of a jet along its axis with the formation of thinner fibers $[23,27,29]$. On the other hand, some studies reported the formation of thicker fibers with the addition of salts due to a large increase in the mass flow [30,31]. This increase can be attributed to the presence of noncovalent interactions between additives and polymers.

Another important parameter in the electrospinning process is the humidity, which has shown a significant impact on the texture of the resultant fibers when a volatile solvent (i.e., tetrahydrofuran (THF) or chloroform $\left(\mathrm{CHCl}_{3}\right)$ ) was used for the dissolution of the polymer. It was reported that the electrospinning of polystyrene (PS), polycarbonate (PC), and poly(methyl methacrylate) (PMMA) from $\mathrm{CHCl}_{3}$ and THF led to the fibers with a porous texture as a result of rapid evaporation of solvent molecules [33]. In the electrospinning of such polymers, increasing relative humidity induced larger pores on the fiber surface. A similar finding was reported by the electrospinning of PS at increasing relative humidity, where the authors observed the formation of larger pores with increasing relative humidity [34]. Since the presence of pores on the fibers enhances the surface area, it is highly desired to produce electrospun membranes from such porous fibers. In this regard, the influence of relative humidity and solution conductivity on the electrospinning of PIMs becomes highly critical. However, to the best of our knowledge, both parameters have not been addressed yet for the electrospinning of PIM-1 fibers.

In this study, the influence of solution conductivity and relative humidity on the electrospinning of PIM-1 was investigated. The influence of the addition of TEAB on the conductivity and viscosity of PIM-1 solutions in different concentrations was explored and thereafter, the respective solutions were electrospun into fibers at various formulations. Likewise, the electrospinning of PIM-1 solutions was performed at different humidity conditions, and the fiber morphology was explored by scanning electron microscopy. The experimental findings were discussed with respect to the influence of humidity and conductivity on the electrospinnability of PIM-1.

\section{Experimental}

\subsection{Materials}

Tetrafluoroterephthalonitrile (TFTN, 98\%, Alfa Aeser) and 5,5',6,6'-

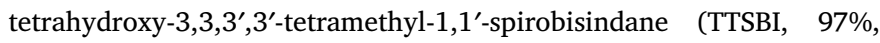
Alfa Aesar) were purified as reported previously [13]. Anhydrous potassium carbonate $\left(\mathrm{K}_{2} \mathrm{CO}_{3}, 99.0 \%\right.$, Alfa Aeser $)$ was grinded and dried in an oven at $110{ }^{\circ} \mathrm{C}$ overnight prior to its use. Chloroform $\left(\mathrm{CHCl}_{3}\right)$, dimethylacetamide (DMAc), toluene, dichloromethane (DCM), methanol $(\mathrm{MeOH})$, and 1,1,2,2-tetrachloroethane (TeCA) were received from Sigma Aldrich. Tetraethylammonium bromide (TEAB, 98\%) was purchased from Alfa Aesar.

\subsection{Synthesis of Polymers of Intrinsic Microporosity (PIM-1)}

The synthesis of PIM-1 was performed according to our previous method [35]. Briefly, TTSBI $(34.04 \mathrm{~g}, 0.1 \mathrm{~mol})$, TFTN $(20.01 \mathrm{~g}$, $0.1 \mathrm{~mol})$, anhydrous $\mathrm{K}_{2} \mathrm{CO}_{3}(41.4 \mathrm{~g}, 0.3 \mathrm{~mol})$, DMAc $(200 \mathrm{~mL})$ and toluene $(100 \mathrm{~mL})$ were reacted in $1 \mathrm{~L}$ three-necked round-bottomed flask equipped with a Dean-Stark trap and mechanical stirrer at $160{ }^{\circ} \mathrm{C}$ for $45 \mathrm{~min}$ under reflux. The resulting highly viscous solid-like polymer was washed with methanol and afterward dissolved in $\mathrm{CHCl}_{3}$ and reprecipitated from $\mathrm{MeOH}$. The polymer was refluxed in deionized water for $4 \mathrm{~h}$ and then, dried at $110^{\circ} \mathrm{C}$ for 2 days. Yield: $42.8 \mathrm{~g}$ (93\%). GPC: $M_{\mathrm{n}}=61000 \mathrm{~g} \mathrm{~mol}^{-1}, M_{\mathrm{w}}=93000 \mathrm{~g} \mathrm{~mol}^{-1}, M_{\mathrm{w}} / M_{\mathrm{n}}=1.52 .{ }^{1} \mathrm{H} \mathrm{NMR}$ (400 MHz, $\left.\mathrm{CDCl}_{3}, \mathrm{~d}, \mathrm{ppm}\right): 6.75(2 \mathrm{H}, \mathrm{s}), 6.35(2 \mathrm{H}, \mathrm{s}), 2.26-2.09(4 \mathrm{H}$, dd), 1.40-1.10 (broad, $12 \mathrm{H}$ ). ATR-IR $\left(\mathrm{cm}^{-1}\right)$ : 2995, 2864, 2239, 1605, 1446, 1264. The composition of PIM-1 according to elemental analysis: (anal. calculated) $\mathrm{C}_{29} \mathrm{H}_{20} \mathrm{~N}_{2} \mathrm{O}_{4}$ (wt\%): C: $75.64, \mathrm{H}: 4.37, \mathrm{~N}: 6.08$, (found) C: $72.2, \mathrm{H}: 4.3, \mathrm{~N}: 6.0$; according to XPS analysis (\% atomic): C: 92.5, N: 3.5, O: 4. The BET surface area of the PIM-1 powder was measured to be $768 \mathrm{~m}^{2} / \mathrm{g}$ while the micropore area was found as 372 $\mathrm{m}^{2} / \mathrm{g}$. All respective results related to the ${ }^{1} \mathrm{H}$ NMR, FTIR, TGA, GPC, and BET were shown in Fig. 1 and Figs. S1-2. 
(a)
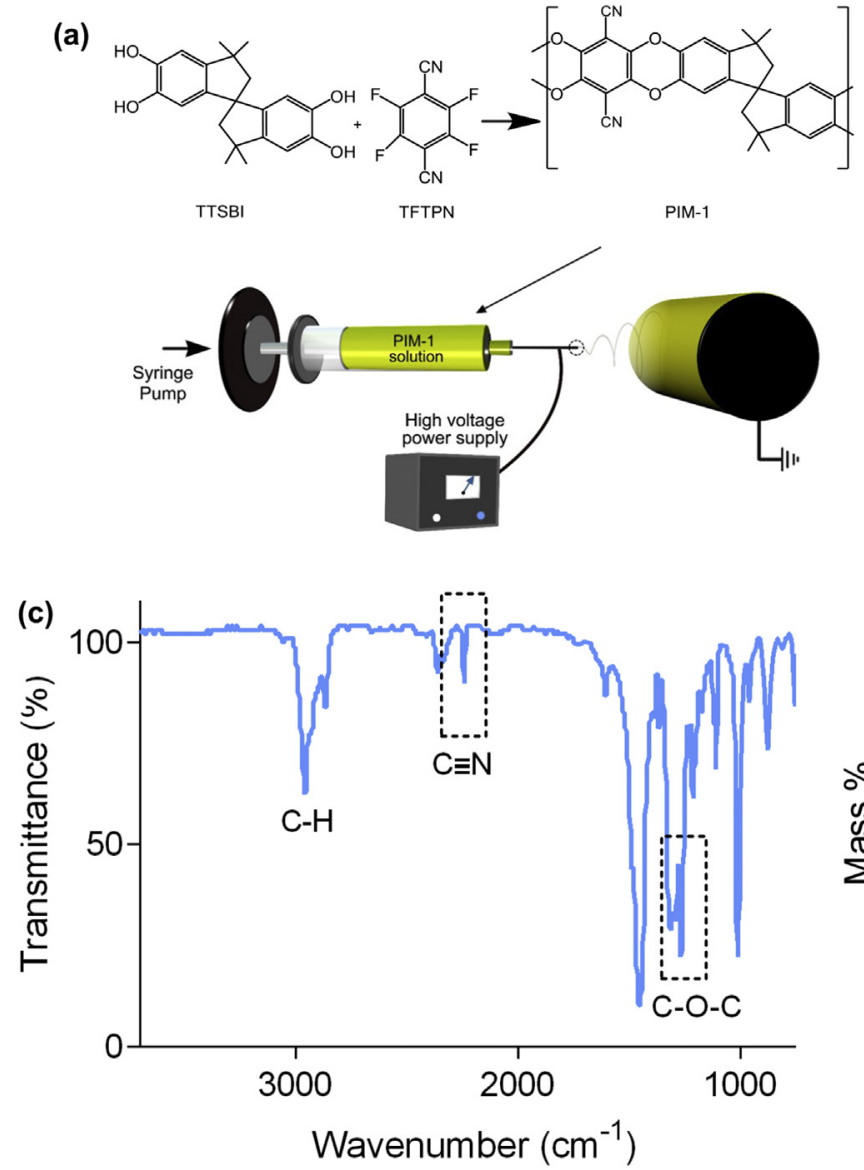

(b)
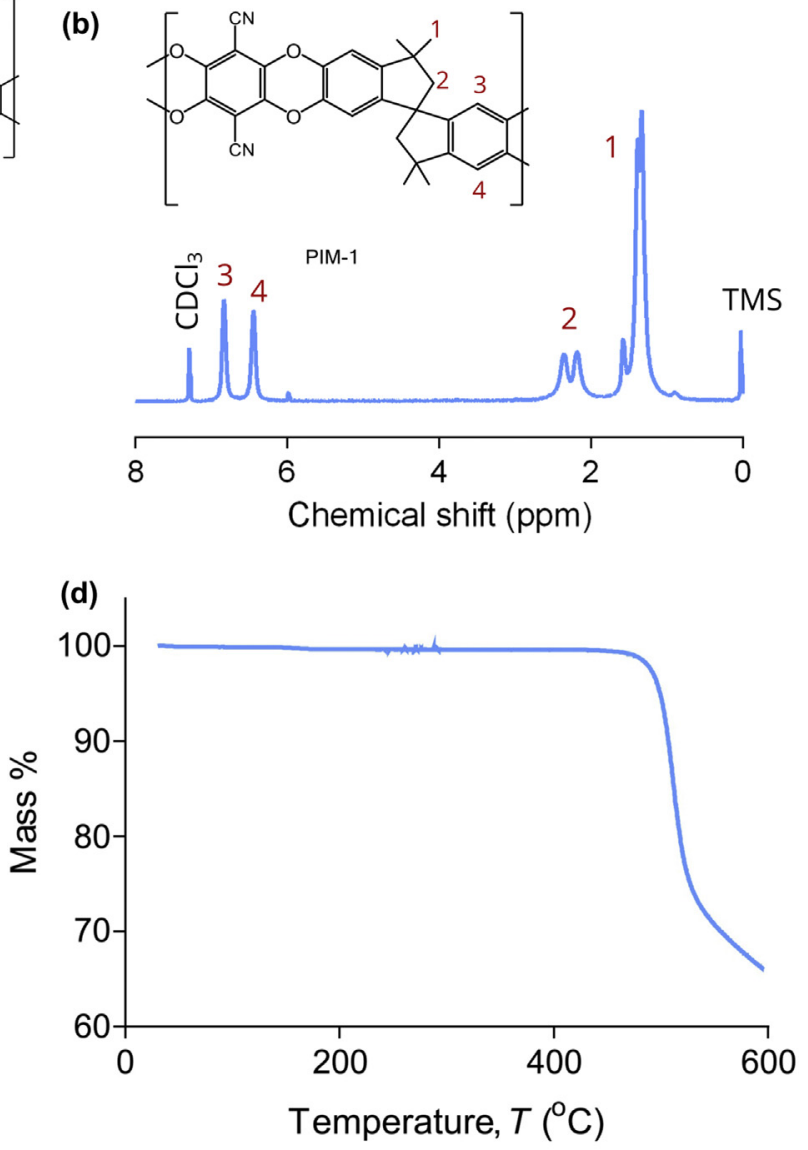

Fig. 1. (a) The synthesis scheme and electrospinning of PIM-1 from its solution in TCE. (b) ${ }^{1}$ H NMR, (c) FTIR and (d) TGA analyses of the PIM-1.

\subsection{Electrospinning of PIM-1 solutions}

PIM-1 powder was dissolved in TeCA under continuous stirring for at least $2 \mathrm{~h}$. Thereafter, the solutions were transferred into $1 \mathrm{~mL}$ plastic disposable syringes with blunt-edged metallic needles (18G Sterican ${ }^{\circ}$ MIX blunt, B.Braun). The syringes were placed on a syringe pump (KD Scientific, KDS 101) and discharged at a certain speed $\left(0.5 \mathrm{~mL} \mathrm{~h}^{-1}\right)$. A rotator collector covered by aluminum foil was used for the deposition of electrospun fibers. During the electrospinning process, process variables held constant: the flow rate of the polymer solution was $0.5 \mathrm{~mL}$ $\mathrm{h}^{-1}$, the applied voltage was $12 \mathrm{kV}$ and the tip-to-collector distance was $20 \mathrm{~cm}$, and the rotation speed of the collector kept at $2000 \mathrm{rpm}$. Afterward, the morphology of the electrospun fibers was explored by SEM analysis.

\subsection{Characterization}

The molecular weight of the PIM-1 was measured with an Agilent gel permeation chromatography (GPC) equipped with a ZORBAX PSM 300 -S column. Polystyrene with different molecular weights was used for the calibration curve, and THF was exploited as a mobile phase. ${ }^{1} \mathrm{H}$ NMR spectrum of the PIM- 1 in $\mathrm{CDCl}_{3}$ was recorded on a Bruker DPX$400 \mathrm{MHz}$ spectrometer, and the data analysis was performed using Mnova (ver. 9, MestreLab). The conductivity of PIM-1 solutions prepared at various salt concentrations was measured by a conductivity meter (Mettler Toledo) at room temperature. The experiments were performed in triplicate, and the mean data with \pm SD were presented. The viscosity of PIM-1 solutions was measured by Anton Paar MCR 301 rheometer using a cone-plate geometry $\left(20 \mathrm{~mm}, 1^{\circ}\right)$ at $25^{\circ} \mathrm{C}$, and the viscosity value at $100 \mathrm{~s}^{-1}$ was given. Fourier transform infrared (FTIR) spectrum of the PIM-1 was recorded on a Bruker-VERTEX 70 spectrometer. The spectrum was taken at a resolution $4 \mathrm{~cm}^{-1}$ after 64 scans accumulation. The porosity of the PIM-1 powder and fibers was measured over $\mathrm{N}_{2}$ sorption isotherms at $77 \mathrm{~K}$ by a Quantachrome Autosorb iQ gas sorption analyzer. The samples were degassed for $24 \mathrm{~h}$ at $120^{\circ} \mathrm{C}$ under vacuum. Thermal analysis of the PIM-1 was performed using a thermogravimetric analyzer (TA Q500, TA Instruments). The XPS spectrum of the PIM-1 was recorded with a Thermo Fisher Scientific Kalpha XPS spectrometer, which is equipped with monochromatic Al Kalpha source. The elemental analysis of the PIM-1 was performed using a Thermo Scientific Flash 2000 series CHNS-O analyzer. 2,5-bis(5tertbutyl-2-benzo-oxazol-2-yl)) thiophene was used as a standard. PIM$1(\sim 1 \mathrm{mg})$ was mixed with a catalyst vanadium pentoxide $(8 \mathrm{mg})$ for complete oxidation The morphology of the fibers and electrosprayed beads was explored using a scanning electron microscopy (Quanta 200 FEG, FEI). Prior to SEM analysis, the specimens were coated $10 \mathrm{~nm} \mathrm{Au}$ with a GATAN PECS ${ }^{\text {TM }}$ sputter coating system. The mean diameter of fibers was calculated over 10-100 fibers from the respective SEM photos using ImageJ analysis software (US National Institutes of Health, Bethesda, Maryland, USA).

\section{Results and discussion}

PIM-1 was successfully synthesized from TTSBI and TFTN by condensation reactions at $160^{\circ} \mathrm{C}$ under reflux (Fig. 1a). After the synthesis, the polymer was characterized by GPC, ${ }^{1} \mathrm{H}$ NMR, FTIR, TGA and BET measurements. ${ }^{1} \mathrm{H}$ NMR analysis of the PIM-1 revealed four different proton peaks, and the ratio of aliphatic (appeared between 0.9 and $2.5 \mathrm{ppm}$ for $\mathrm{H} 1$ and $\mathrm{H} 2$ ) and aromatic (appeared between 6 and $7 \mathrm{ppm}$ for $\mathrm{H} 3$ and $\mathrm{H} 4$ ) protons were found to be 4, demonstrating the 

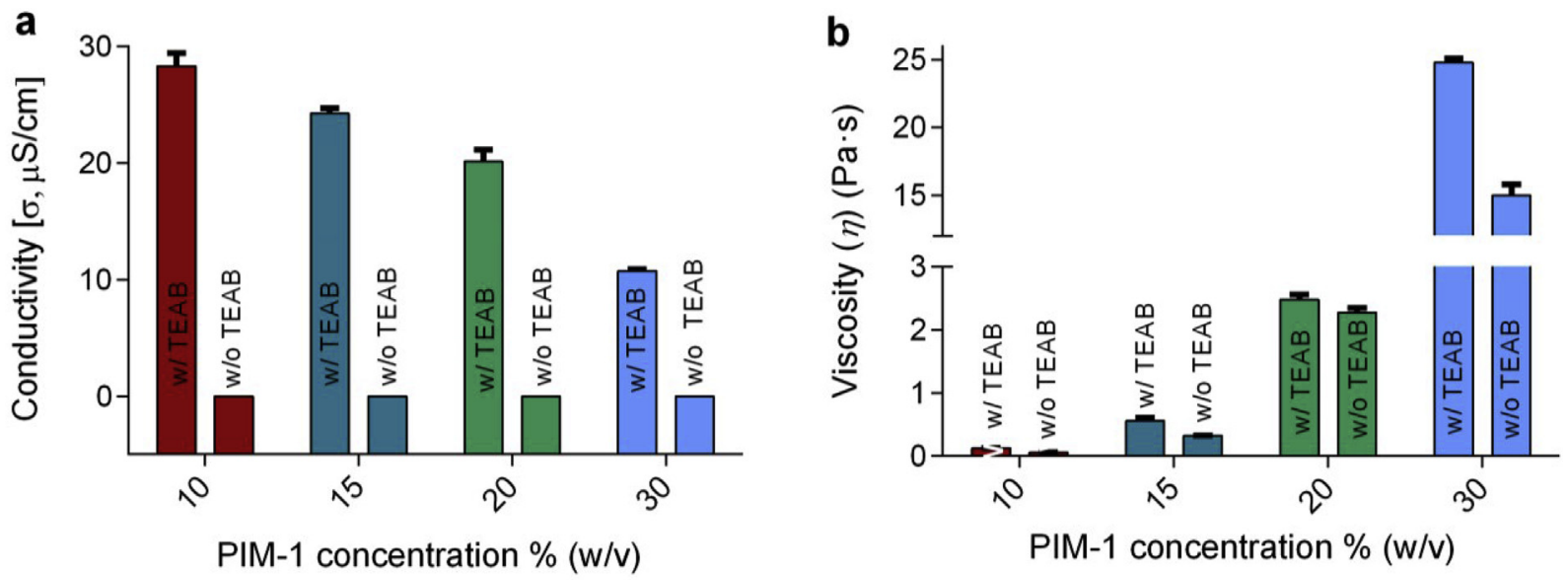

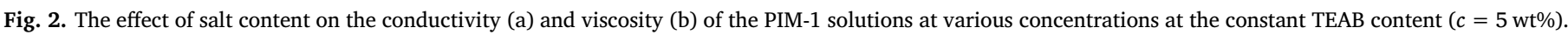

successful synthesis of PIM-1 (Fig. 1b) [36]. Likewise, the FTIR spectra of the PIM-1 showed characteristic ether and nitrile stretches at 1265 and $2240 \mathrm{~cm}^{-1}$ while aliphatic and aromatic C-H stretches appeared in the region of $2800-3010 \mathrm{~cm}^{-1}$ (Fig. 1c). TGA analysis revealed the intrinsic thermal stability of PIM-1 up to $460{ }^{\circ} \mathrm{C}$ (Fig. 1d). The molecular weight $\left(M_{\mathrm{w}}\right)$ of the PIM-1 was measured as $93000 \mathrm{~g} \mathrm{~mol}^{-1}$, and $M_{\mathrm{w}} / M_{\mathrm{n}}$ was found to be 1.52 (Fig. S1), while the BET surface area of the PIM-1 was measured to be $768 \mathrm{~m}^{2} \mathrm{~g}^{-1}$ (Fig. S2). After the electrospinning, no significant change was observed on the BET surface area of PIM-1 (Fig. S2), demonstrating that the electrospinning process did not influence the intrinsic porosity of the polymer and the solvent was eveporated from the polymer matrix.

The electrospinning of PIM-1 was performed from its solution in TeCA in the presence and absence of TEAB to elucidate the influence of TEAB addition on the electrospinnability of PIM-1 solutions. Prior to the electrospinning process, the respective solutions were characterized by conductivity and viscosity measurements to understand the effect of TEAB addition on the properties of PIM-1 solutions. In this regard, Fig. 2a shows the conductivity of PIM-1 solutions in the presence of the constant TEAB content ( $5 \mathrm{wt} \%$ according to the PIM-1 content) at various PIM-1 concentrations (10-30\% (w/v)). Regardless of PIM-1 concentration used, the conductivity of PIM-1 solutions in TeCA was very low (i.e., $<1 \mu \mathrm{S} / \mathrm{cm}$ ) (Fig. 2a), suggesting that both PIM-1 and TeCA have poor conductivity. However, after the addition of $5 \mathrm{wt} \%$ $\mathrm{TEAB}$, the conductivity of the solutions significantly increased. On the other hand, lower conductivity values were observed at increasing PIM1 content in the presence of $5 \mathrm{wt} \%$ TEAB, suggesting that the use of a higher PIM-1 content decreases the solution conductivity. The conductivity of PIM- 1 of $10 \%(\mathrm{w} / \mathrm{v})$ in the presence of $5 \mathrm{wt} \%$ TEAB was measured $28.3 \pm 1.11 \mu \mathrm{S} / \mathrm{cm}$ while it decreased to $10.74 \pm 0.13 \mu \mathrm{S} /$ $\mathrm{cm}$ with increasing PIM-1 concentration to $30 \%(\mathrm{w} / \mathrm{v})$. An increasing trend was observed in the viscosity of PIM-1 solutions with the incorporation of TEAB (Fig. 2b): the viscosity of PIM-1 solutions increased with the TEAB addition for all concentrations of PIM-1 tested. For instance, the viscosity of $10 \%$ (w/v) PIM-1 increased from $0.058 \pm 0.001$ to $0.12 \pm 0.003 \mathrm{~Pa}$ s with the addition of $5 \mathrm{wt} \%$ TEAB (with respect to PIM-1). Higher viscosity was observed with the increasing concentration of PIM-1: the viscosity of PIM-1 solution rose from $0.058 \pm 0.001$ to $14.99 \pm 0.80$ Pa s with increasing PIM-1 concentration from 10 to $30 \%$. An increase in viscosity with the TEAB addition can be attributed to the formation of intermolecular interactions between TEAB and PIM-1, which led to a rise in the viscosity of the respective mixtures (Fig. $2 \mathrm{~b}$ ).

The electrospinning of PIM-1 solutions was achieved at various concentrations in the presence and absence of $5 \mathrm{wt} \%$ TEAB. The SEM images of the respective fibers were shown in Fig. 3. Regardless of TEAB presence, at higher PIM-1 concentrations, bead-free electrospun fibers were observed, where the mean fiber diameter was above $1000 \mathrm{~nm}$, demonstrating the formation of PIM-1 microfibers. However, decreasing PIM-1 concentration reduced the fiber diameter and led to the beaded-nanofibers at the PIM-1 concentration of $15 \%(\mathrm{w} / \mathrm{v})$. On the other hand, the addition of $5 \mathrm{wt} \%$ TEAB led to bead-free fiber structure, and the mean fiber diameter increased from $590 \pm 140$ to $680 \pm 120 \mathrm{~nm}$, suggesting the formation of thicker fibers with the disappearance of beads on the fibers. The co-existence of splashing and electrosprayed beads was observed when PIM-1 concentration was $10 \%$ $(\mathrm{w} / \mathrm{v})$. This concentration of PIM-1 was not enough to be electrospun into fibers. However, upon addition of $5 \mathrm{wt} \% \mathrm{TEAB}$, quite thin nanofibers were observed. In parallel to the bead-free nanofibers, the formation of some beaded nanofibers was also observed. The mean diameter of the nanofibers was calculated to be $240 \pm 90 \mathrm{~nm}$. In general, the addition of TEAB slightly increased the fiber diameter, which could be attributed to the presence of intermolecular interactions between TEAB and PIM-1. The incorporation of TEAB increased the conductivity of PIM-1 solution, which facilitated the electrospinnability of PIM-1.

The electrospinnability of PIM-1 solutions was also explored at the constant PIM-1 concentration and various TEAB contents. The conductivity values of the respective solutions were shown in Fig. 4a, where the concentration of PIM- 1 held constant at $10 \%(\mathrm{w} / \mathrm{v})$ while the TEAB concentration was increased from 1 to $15 \mathrm{wt} \%$ with respect to the PIM-1 content. With increasing TEAB concentration, the conductivity of PIM-1 solutions significantly enhanced: the PIM-1 solution with $1 \mathrm{wt} \%$ TEAB has conductivity value of $5.09 \pm 0.06 \mu \mathrm{S} / \mathrm{cm}$ while the respective value for the PIM-1 solution with $15 \mathrm{wt} \%$ is $90.03 \pm 0.153 \mu \mathrm{S} / \mathrm{cm}$, demonstrating the drastic effect of TEAB presence on the conductivity of PIM-1 solutions. On the other hand, the viscosity of the respective mixtures was also explored. Fig. 4b shows the viscosity values of the PIM- 1 solutions $(c=10 \%(\mathrm{w} / \mathrm{v}))$ in the presence of various TEAB contents (1-15 wt\%), where the viscosity showed an increasing trend with a TEAB content rise up to $5 \mathrm{wt} \%$, and thereafter, the viscosity decreased to values comparable to the TEAB-free solution. The increase in viscosity can be attributed to the existence of possible interactions between the PIM backbone and amphiphilic tetraethylammonium salt, which increased the viscosity of the respective mixtures: particularly, nitrile groups in the lateral position of the PIM-1 backbone might readily interact with the quaternary nitrogen group in the TEAB. Whereas, with a further rise in the TEAB content, the viscosity of the respective solutions decreases to some extent.

The influence of TEAB content at the constant PIM-1 concentration $(10 \%(\mathrm{w} / \mathrm{v}))$ was explored over the electrospinnability of the respective mixtures. Fig. 5 displays the morphology of the electrospun fibers of the respective solutions. In the absence of TEAB, both electrospraying and splashing took place at the identical concentration of PIM-1. Whereas the incorporation of $1 \mathrm{wt} \%$ TEAB into the PIM-1 solutions led to 

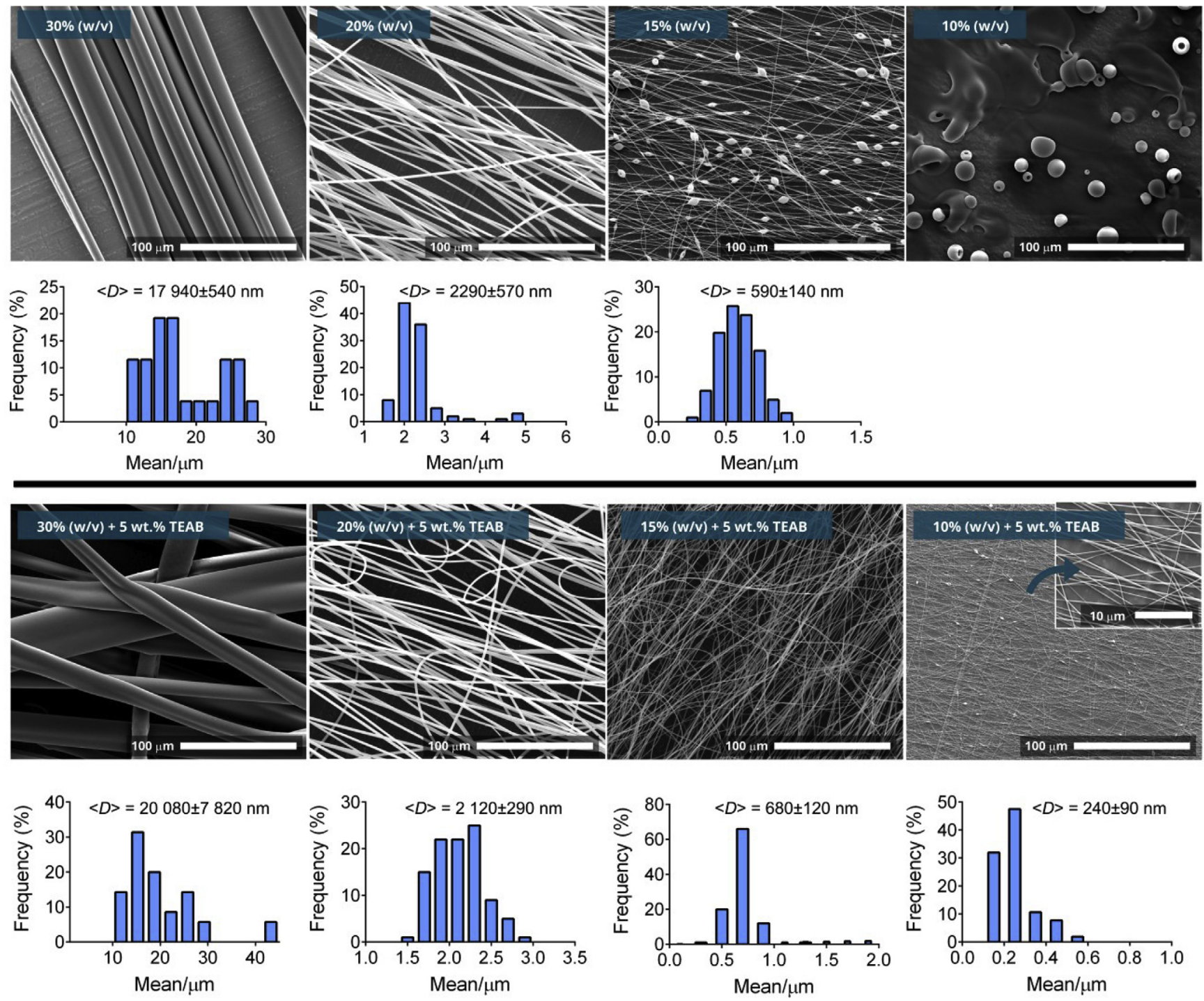

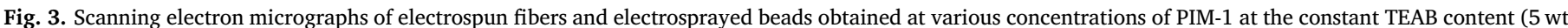

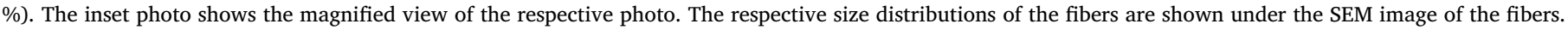

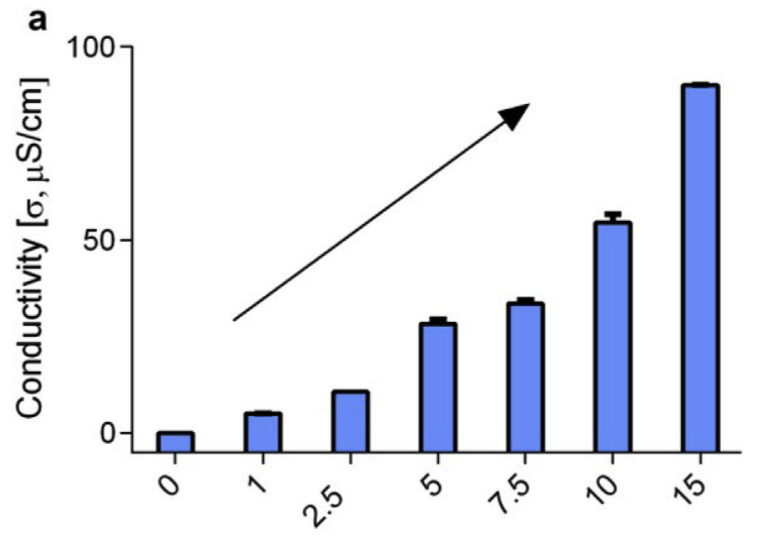

TEAB concentration (wt.\%)

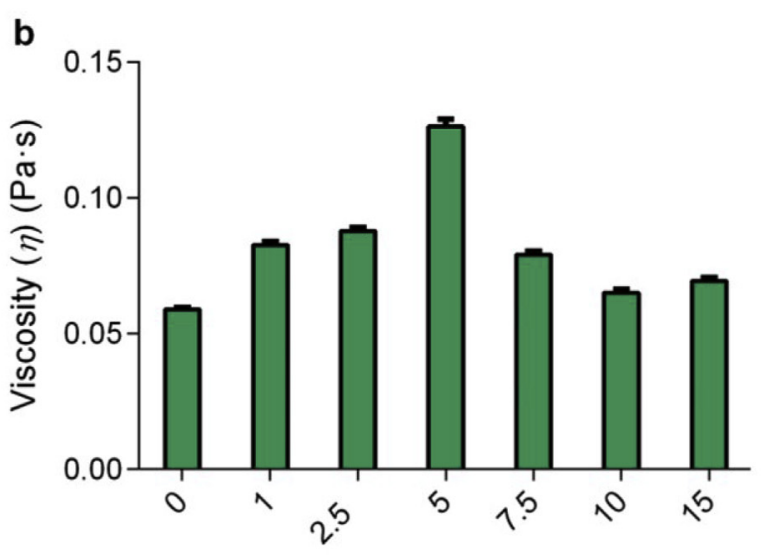

TEAB concentration (wt.\%)

Fig. 4. The influence of TEAB content on the conductivity (a) and viscosity (b) of the PIM-1 solutions containing various salt concentrations. 


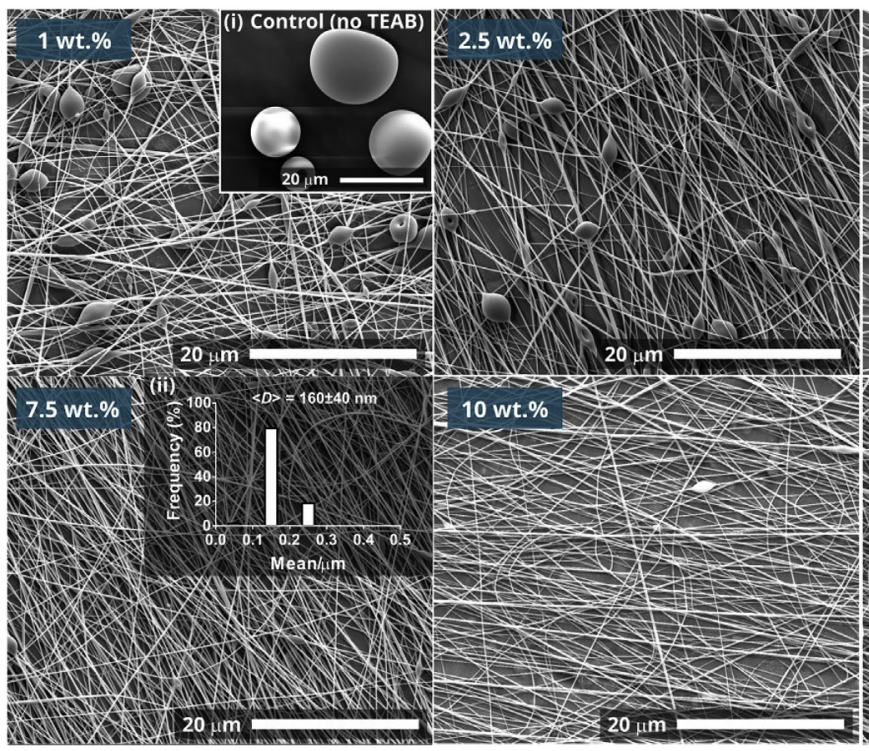

beaded-fibers. This drastic difference can be accredited to the decrease of the jet diameter with the TEAB presence before reaching to the collector plate. On the other hand, increasing the TEAB concentration decreased the number of beads formed on the fibers. In the presence of $7.5 \mathrm{wt} \%$ TEAB, very thin PIM-1 fibers with $160 \pm 40 \mathrm{~nm}$ in diameter were obtained while the resultant fibers have nearly bead-free fiber structure. To the best of the authors' knowledge, this is the lowest diameter for the electrospun PIM-1 fibers reported to date. In this regard, Ding et al. reported the formation of thinner fibers for poly(vinyl alcohol) nanofibers with the addition of $\mathrm{NaCl}$ and $\mathrm{Fe}\left(\mathrm{NO}_{3}\right)_{3}$ [37]. However, a contradictory effect of salt was also observed for the diameters of polyvinylpyrrolidone fibers, where the fiber diameter increased with the addition of salts [38]. Likewise, the addition of salt increased the viscosity of the respective solutions, and hence, the formation of thicker fibers can be attributed to the enhanced viscosity, i.e., a higher mass flow during the electrospinning process.

In addition to the effect of conductivity, the influence of relative humidity on the electrospinning of PIM-1 fibers was investigated. In this regard, the electrospinning of PIM-1 solutions was conducted at the concentration of $25 \mathrm{wt} \%$ which enables the formation of bead-free fibers without the necessity of any additive/salt and at various humidity conditions between 25 and $80 \%$ while keeping other parameters constant (Fig. 6). For all humidity conditions, bead-free fiber structure was obtained, and the effect of humidity on the fiber diameters was insignificant. For instance, the mean diameter of the fibers electrospun at $25 \%$ humidity was $2280 \pm 480 \mathrm{~nm}$ while it slightly increased $2390 \pm 380 \mathrm{~nm}$ with increasing humidity to $80 \%$. On the other hand, fewer fibers were formed at very high humidity conditions $(\sim 80 \%)$, which may be attributed to a considerable loss of charge from the spinning head. Furthermore, the effect of relative humidity was also explored on the texture of the respective fibers at higher magnifications. Fig. S3 shows no significant change on the texture of the fibers produced at various humidity conditions, suggesting that humidity does not have any profound influence on the texture and diameters of the resultant fibers. The mean fiber diameter did not reveal any drastic difference depending on relative humidity (Fig. S4).

The effect of humidity was also explored at a lower PIM concentration (i.e., $15 \%(\mathrm{w} / \mathrm{v})$ ) for better understanding the influence of relative humidity on the electrospinning of PIM-1 solutions. The electrospinning of PIM-1 at $15 \%$ (w/v) led to electrosprayed beads and beaded-fibers (Fig. S5) while splashing took place at $80 \%$ relative humidity, demonstrating that extreme humidity conditions are not good for the electrospinning of PIM-1. The experimental findings suggest that

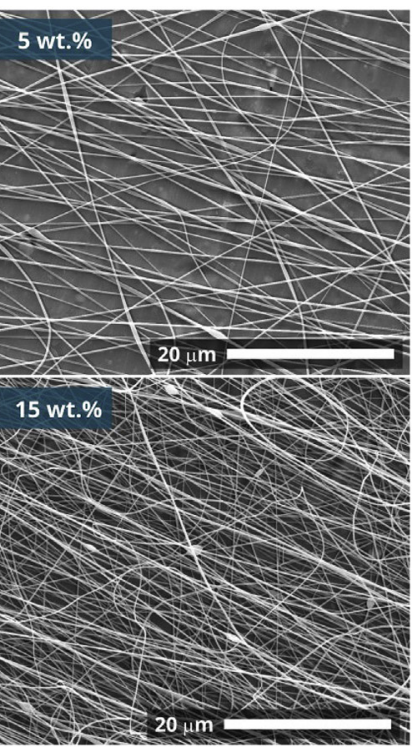

Fig. 5. Scanning electron micrographs of PIM-1 fibers produced in the presence of various TEAB concentrations. Inset (i) shows the SEM photo of the electrosprayed/ splashed beads, which were observed in the absence of TEAB. $c_{\mathrm{PIM}-1}=10 \%(\mathrm{w} / \mathrm{v})$. Inset (ii) shows the size distribution of the respective fibers. For other fibers, the size distribution of the fibers was not shown owing to their beaded-fiber structure. there is no drastic influence of relative humidity on the electrospinning and texture of PIM-1 fibers.

The electrospinnability of PIM-1 solutions from TeCA is highly dependent on the conductivity and suffers from poor conductivity of the respective mixtures. Hence, a higher threshold concentration of PIM-1 is required to able spun into bead-free fibers. However, this causes the formation of very thick fibers $(>2000 \mathrm{~nm})$. On the other hand, the incorporation of TEAB drastically improved the electrospinnability and could lead to bead-free fibers at the low PIM-1 concentrations. The influence of TEAB on the solution property of PIM-1 can be elucidated by both enhanced conductivity and interactions between TEAB and PIM-1. The previous studies regarding the electrospinning of PIM-1 have reported that the mean diameters of the fibers were only achieved above $1 \mu \mathrm{m}[13,15,18]$. In the present study, we could obtain very thin PIM-1 nanofibers with a mean diameter as low as $160 \mathrm{~nm}$. To the best of the authors' knowledge, this study, for the first time, reports the production of the thinnest nanofibers reported for all PIM types in literature.

\section{Conclusion}

The effect of conductivity and relative humidity on the electrospinning of PIM-1 solutions from TeCA was studied. The experimental findings showed that the electrospinnability of PIM-1 solutions is directly related to the conductivity of the respective solutions. The addition of ammonium salt (TEAB) drastically increased the conductivity of PIM-1 solutions and led to the formation of fibers at lower PIM-1 concentrations $(10 \%(\mathrm{w} / \mathrm{v}))$ : higher conductivity could facilitate the jet ejection and therefore, the fibers could be obtained at lower PIM-1 concentrations. The addition of TEAB slightly increased the mean fiber diameter, which was also supported by a rise in the viscosity of the respective solutions. On the other hand, the effect of humidity on the electrospinning of PIM-1 was negligible, and no drastic difference in the morphology and diameter of the fibers was observed. However, at very high humidity conditions, less fibers were formed in a given time. Overall, this paper, for the first time, reports the effect of conductivity and relative humidity on the electrospinnability of PIM-1 solutions and shows the formation of ultrafine PIM- 1 nanofibers with the addition of TEAB.

\section{Conflicts of interest}

The authors declare no conflict of interest. 

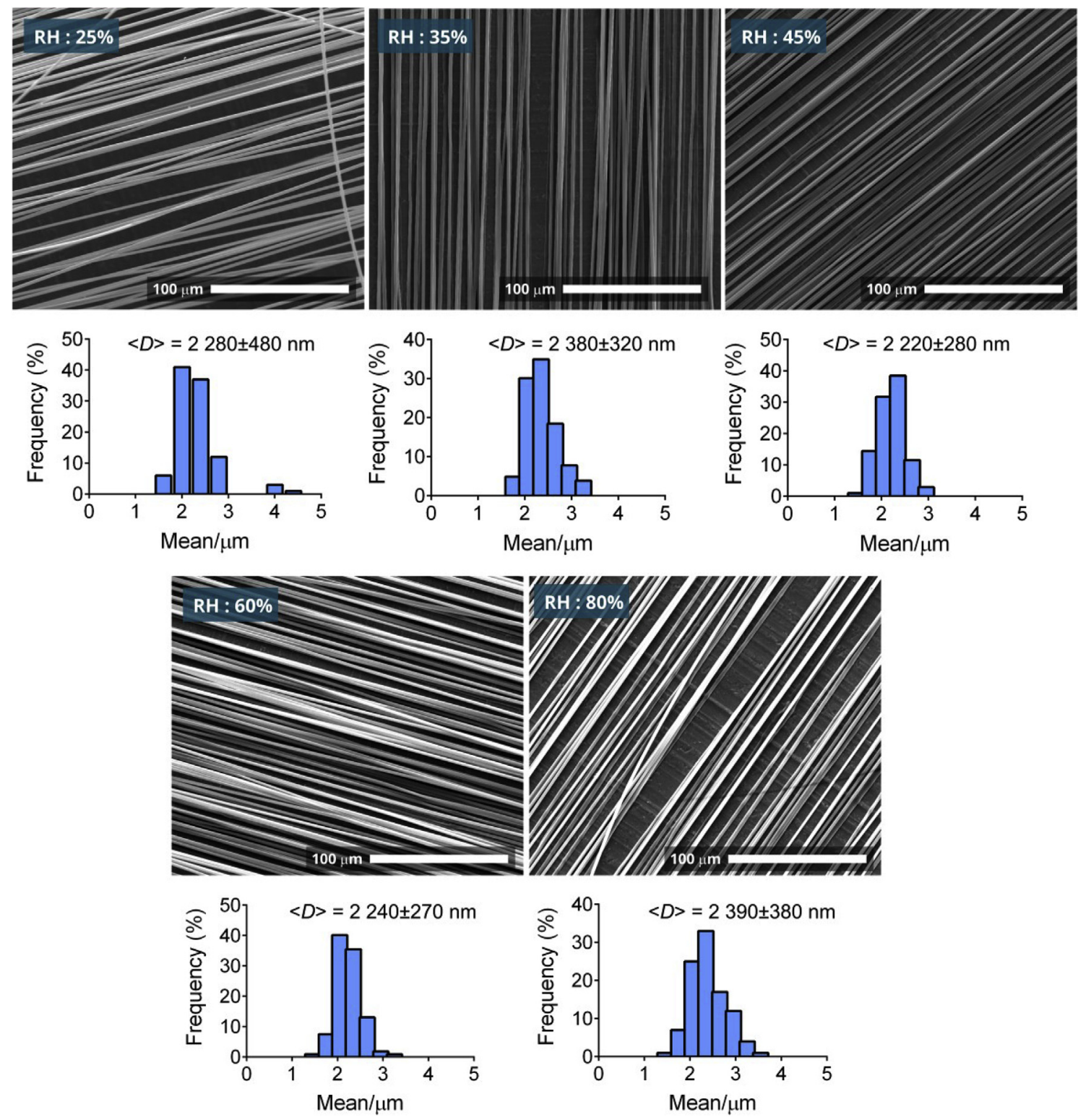

Fig. 6. The influence of relative humidity on the electrospinning of PIM-1 fibers ( $c=25 \%(\mathrm{w} / \mathrm{v})$ at various humidity conditions (25-80\%). SEM images and size distribution of the fibers produced at various relative humidity conditions.

\section{Appendix A. Supplementary data}

Supplementary data to this article can be found online at https:// doi.org/10.1016/j.polymer.2019.121610.

\section{References}

[1] S. Chen, S. Tang, Y. Sun, G. Wang, H. Chen, X. Yu, Y. Su, G. Chen, Preparation of a highly porous carbon material based on quinoa husk and its application for removal of dyes by adsorption, Materials (Basel, Switzerland) 11 (8) (2018) 1407.

[2] A. Alsbaiee, B.J. Smith, L. Xiao, Y. Ling. D. F. Helbling W R. Dichtel, Rapid removal of organic micropollutants from water by a porous $\beta$-cyclodextrin polymer, Nature 529 (2015) 190

[3] R. Shen, X. Yan, Y.-J. Guan, W. Zhu, T. Li, X.-G. Liu, Y. Li, Z.-G. Gu, One-pot synthesis of a highly porous anionic hypercrosslinked polymer for ultrafast adsorption of organic pollutants, Polym. Chem. 9 (38) (2018) 4724-4732.

[4] J. He, J. Dai, T. Zhang, J. Sun, A. Xie, S. Tian, Y. Yan, P. Huo, Preparation of highly porous carbon from sustainable $\alpha$-cellulose for superior removal performance of tetracycline and sulfamethazine from water, RSC Adv. 6 (33) (2016) 28023-28033.

[5] N.B. McKeown, P.M. Budd, Polymers of Intrinsic Microporosity, Encyclopedia of Polymer Science and Technology, John Wiley \& Sons, Inc, 2009.

[6] P.M. Budd, N.B. McKeown, D. Fritsch, Free volume and intrinsic microporosity in polymers, J. Mater. Chem. 15 (20) (2005) 1977-1986.
[7] N.B. McKeown, P.M. Budd, Polymers of intrinsic microporosity (PIMs): organic materials for membrane separations, heterogeneous catalysis and hydrogen storage, Chem. Soc. Rev. 35 (8) (2006) 675-683.

[8] P.M. Budd, B.S. Ghanem, S. Makhseed, N.B. McKeown, K.J. Msayib, C.E. Tattershall, Polymers of intrinsic microporosity (PIMs): robust, solution-pro cessable, organic nanoporous materials, Chem. Commun. (2) (2004) 230-231.

[9] D. Ramimoghadam, E.M. Gray, C.J. Webb, Review of polymers of intrinsic microporosity for hydrogen storage applications, Int. J. Hydrogen Energy 41 (38) (2016) 16944-16965.

[10] N.B. McKeown, The synthesis of polymers of intrinsic microporosity (PIMs), Sci. China Chem. 60 (8) (2017) 1023-1032.

[11] Synthesis and gas permeation properties of spirobischromane-based polymers of intrinsic microporosity, Macromol. Chem. Phys. 212 (11) (2011) 1137-1146.

[12] P. Gorgojo, S. Karan, H.C. Wong, M.F. Jimenez-Solomon, J.T. Cabral, A.G. Livingston, Ultrathin polymer films with intrinsic microporosity: anomalous solvent permeation and high flux membranes, Adv. Funct. Mater. 24 (30) (2014) $4729-4737$.

[13] B. Satilmis, T. Uyar, Removal of aniline from air and water by polymers of intrinsic microporosity (PIM-1) electrospun ultrafine fibers, J. Colloid Interface Sci. 516 (2018) 317-324.

[14] X.M. Wu, Q.G. Zhang, F. Soyekwo, Q.L. Liu, A.M. Zhu, Pervaporation removal of volatile organic compounds from aqueous solutions using the highly permeable PIM-1 membrane, AIChE J. 62 (3) (2016) 842-851.

[15] C. Zhang, P. Li, B. Cao, Electrospun polymer of intrinsic microporosity fibers and their use in the adsorption of contaminants from a nonaqueous system, J. Appl. 
Polym. Sci. 133 (22) (2016).

[16] C. Zhang, P. Li, B. Cao, Electrospun microfibrous membranes based on PIM-1/POSS with high oil wettability for separation of oil-water mixtures and cleanup of oil soluble contaminants, Ind. Eng. Chem. Res. 54 (35) (2015) 8772-8781.

[17] J.S. Bonso, G.D. Kalaw, J.P. Ferraris, High surface area carbon nanofibers derived from electrospun PIM-1 for energy storage applications, J. Mater. Chem. 2 (2) (2014) 418-424.

[18] K. Halder, G. Bengtson, V. Filiz, V. Abetz, Catalytically active (Pd) nanoparticles supported by electrospun PIM-1: influence of the sorption capacity of the polymer tested in the reduction of some aromatic nitro compounds, Appl. Catal. Gen. 555 (2018) 178-188.

[19] C. Zhang, P. Li, W. Huang, B. Cao, Selective adsorption and separation of organic dyes in aqueous solutions by hydrolyzed PIM-1 microfibers, Chem. Eng. Res. Des. 109 (2016) 76-85.

[20] P. Gupta, C. Elkins, T.E. Long, G.L. Wilkes, Electrospinning of linear homopolymers of poly(methyl methacrylate): exploring relationships between fiber formation, viscosity, molecular weight and concentration in a good solvent, Polymer 46 (13) (2005) 4799-4810.

[21] A. Koski, K. Yim, S. Shivkumar, Effect of molecular weight on fibrous PVA produced by electrospinning, Mater. Lett. 58 (3) (2004) 493-497.

[22] D.H. Reneker, A.L. Yarin, Electrospinning jets and polymer nanofibers, Polymer 49 (10) (2008) 2387-2425.

[23] T. Uyar, F. Besenbacher, Electrospinning of uniform polystyrene fibers: the effect of solvent conductivity, Polymer 49 (24) (2008) 5336-5343.

[24] Y.-Z. Chen, P. Peng, Z.-X. Guo, J. Yu, M.-S. Zhan, Effect of hyperbranched poly(ester amine) additive on electrospinning of low concentration poly(methyl methacrylate) solutions, J. Appl. Polym. Sci. 115 (6) (2010) 3687-3696.

[25] X. Wang, C. Pellerin, C.G. Bazuin, Enhancing the electrospinnability of low molecular weight polymers using small effective cross-linkers, Macromolecules 49 (3) (2016) 891-899.

[26] H. Fong, I. Chun, D.H. Reneker, Beaded nanofibers formed during electrospinning, Polymer 40 (16) (1999) 4585-4592.

[27] S.H. Tan, R. Inai, M. Kotaki, S. Ramakrishna, Systematic parameter study for ultra- fine fiber fabrication via electrospinning process, Polymer 46 (16) (2005) 6128-6134.

[28] X. Zong, K. Kim, D. Fang, S. Ran, B.S. Hsiao, B. Chu, Structure and process relationship of electrospun bioabsorbable nanofiber membranes, Polymer 43 (16) (2002) 4403-4412.

[29] W.K. Son, J.H. Youk, T.S. Lee, W.H. Park, The effects of solution properties and polyelectrolyte on electrospinning of ultrafine poly(ethylene oxide) fibers, Polymer 45 (9) (2004) 2959-2966.

[30] C. Mit-uppatham, M. Nithitanakul, P. Supaphol, Ultrafine electrospun polyamide-6 fibers: effect of solution conditions on morphology and average fiber diameter, Macromol. Chem. Phys. 205 (17) (2004) 2327-2338.

[31] A.K. Moghe, R. Hufenus, S.M. Hudson, B.S. Gupta, Effect of the addition of a fugitive salt on electrospinnability of poly(e-caprolactone), Polymer 50 (14) (2009) 3311-3318.

[32] L. Li, Y.-L. Hsieh, Ultra-fine polyelectrolyte fibers from electrospinning of poly (acrylic acid), Polymer 46 (14) (2005) 5133-5139.

[33] S. Megelski, J.S. Stephens, D.B. Chase, J.F. Rabolt, Micro- and nanostructured surface morphology on electrospun polymer fibers, Macromolecules 35 (22) (2002) 8456-8466.

[34] C.L. Casper, J.S. Stephens, N.G. Tassi, D.B. Chase, J.F. Rabolt, Controlling surface morphology of electrospun polystyrene Fibers: effect of humidity and molecular weight in the electrospinning process, Macromolecules 37 (2) (2004) 573-578.

[35] B. Satilmis, P.M. Budd, T. Uyar, Systematic hydrolysis of PIM-1 and electrospinning of hydrolyzed PIM-1 ultrafine fibers for an efficient removal of dye from water, React. Funct. Polym. 121 (2017) 67-75.

[36] B. Satilmis, P.M. Budd, Base-catalysed hydrolysis of PIM-1: amide versus carboxylate formation, RSC Adv. 4 (94) (2014) 52189-52198.

[37] W. Ding, S. Wei, J. Zhu, X. Chen, D. Rutman, Z. Guo, Manipulated electrospun PVA nanofibers with inexpensive salts, Macromol. Mater. Eng. 295 (10) (2010) 958-965.

[38] K. Nartetamrongsutt, G.G. Chase, The influence of salt and solvent concentrations on electrospun polyvinylpyrrolidone fiber diameters and bead formation, Polymer 54 (8) (2013) 2166-2173. 